Groups Geom. Dyn. 5 (2011), 683-690

DOI $10.4171 / \mathrm{GGD} / 144$
Groups, Geometry, and Dynamics

(C) European Mathematical Society

\title{
Homological and homotopical higher-order filling functions
}

\author{
Robert Young
}

Abstract. We construct groups in which $F V^{3}(n) \nsim \delta^{2}(n)$. This construction also leads to groups $G_{k}, k \geq 3$, for which $\delta^{k}(n)$ is not subrecursive.

Mathematics Subject Classification (2010). 20F65, 57M07.

Keywords. Dehn functions, filling invariants.

The Dehn function of a group provides a measure of the complexity of the group's word problem by measuring the difficulty of filling loops in a corresponding complex. A natural generalization is to consider the difficulty of filling higher-dimensional manifolds or cycles, and there are several ways to do so, varying in the nature of the filling and the boundary. One can consider, for example, the volume necessary to fill a $k$-sphere with a ball $\left(\delta^{k}\right)$, to fill $\partial M$ with $M\left(\delta^{M}\right)$, or to fill a $(k-1)$-cycle by a $k$-chain $\left(F V^{k}\right)$. In some cases, these functions are equivalent; for example, the methods used in [11] work for all these definitions. Along these lines, Brady et al. [3] showed that if $\partial M$ is connected and $\operatorname{dim} M=k+1 \geq 4$ then $\delta^{M}(n) \preceq \delta^{k}(n)$. In this note, we will show that this is not necessarily true if $\operatorname{dim} M=3$, and that there are groups where $F V^{3}$ is not equivalent to $\delta^{2}$. We will also show that for $k \geq 2$ there are groups where $F V^{k}$ is not subrecursive (i.e., $F V^{k}$ grows faster than any computable function) and for $k \geq 3$, there are groups where $\delta^{k}$ is not subrecursive.

We start by defining some filling functions. To define $\delta^{k}$, we will take the approach of Brady et al. [3], which is equivalent to the definition of Alonso, Wang, and Pride [2] or of Bridson [5]. We recall their definition of an admissible map:

Definition 1 (Admissible maps [3]). Let $W$ be a compact $k$-manifold and $X$ a CWcomplex. An admissible map from $W$ to $X$ is a map $f: W \rightarrow X^{(k)}$ such that $f^{-1}\left(X^{(k)} \backslash X^{(k-1)}\right)$ is a disjoint union of open $k$-dimensional balls in $W$, each mapped homeomorphically to a $k$-cell of $X$. We define the volume $\operatorname{vol}(f)$ of $f$ as the number of these balls.

If $\alpha=\sum a_{i} \Delta_{i} \in C_{k}(X ; \mathbb{R})$ is a cellular chain in $X$, with $a_{i} \in \mathbb{R}$ and $\Delta_{i}$ distinct cells of $X$, define $\|\alpha\|_{1}=\sum\left|a_{i}\right|$. If $W$ is orientable and $f: W \rightarrow X$ is an admissible 
map, the image of the fundamental class of $W$ is a cellular $k$-chain, which we call $\hat{f}$. This has integer coefficients, and $\|\hat{f}\|_{1} \leq$ vol $f$. Furthermore, if $W$ is closed, then $\hat{f}$ is a cycle.

If $X$ is a $k$-connected CW-complex, one can define the filling volume of an admissible map $\alpha: S^{k} \rightarrow X$ as

$$
\delta_{X}^{k}(\alpha)=\inf \left\{\operatorname{vol} \beta\left|\beta: D^{k+1} \rightarrow X, \beta\right|_{S^{k}}=\alpha, \beta \text { is admissible }\right\}
$$

and the $k$-th order Dehn function of the complex by

$$
\delta_{X}^{k}(n)=\sup _{\substack{\alpha: S^{k} \rightarrow X \\ \operatorname{vol} \alpha \leq n}} \delta_{X}^{k}(\alpha),
$$

where $\alpha$ is assumed to be admissible.

We can also define the Dehn function of a group:

Definition 2 (Dehn functions). We say a group $G$ is $\mathscr{F}^{k}$ if there is a $K(G, 1)$ with finite $k$-skeleton. If $G$ is $\mathscr{F}^{k+1}$, let $X$ be the $(k+1)$-skeleton of the universal cover of such a $K(G, 1)$ and define the $k$-th order Dehn function of $G$

$$
\delta_{G}^{k}(n)=\delta_{X}^{k}(n)
$$

This function depends on the choice of $X$, but Gromov's Filling Theorem [4] states that the growth rate of $\delta_{X}^{1}$ is an invariant of $G$, and Alonso, Wang, and Pride generalized this to higher dimensions [2]. That is, we define the partial ordering,

$$
\begin{aligned}
& f \preceq g \text { iff there exists } A, B, C, D, E \text { such that } \\
& \qquad f(n) \leq A g(B n+C)+D n+E \text { for all } n>0,
\end{aligned}
$$

and let $f \sim g$ if and only if $f \preceq g$ and $f \succeq g$. If $X_{1}$ and $X_{2}$ are as in Definition 2, then $\delta_{X_{1}}^{k} \sim \delta_{X_{2}}^{k}$.

There are several ways to generalize this beyond fillings of spheres by balls. Brady et al. [3] provide one generalization. If $(M, \partial M)$ is a (smooth or PL) compact manifold pair with $\operatorname{dim} M=k+1$, define the filling volume of an admissible map $\alpha: \partial M \rightarrow X$ as

$$
\delta_{X}^{M}(\alpha)=\inf \left\{\operatorname{vol}(\beta)|\beta: M \rightarrow X, \beta|_{\partial M}=\alpha, \beta \text { is admissible }\right\}
$$

and

$$
\delta_{X}^{M}(n)=\sup _{\substack{\alpha: \partial M \rightarrow X \\ \operatorname{vol} \alpha \leq n}} \delta_{X}^{M}(\alpha),
$$

where $\alpha$ is again assumed to be admissible. In particular, $\delta_{X}^{D^{k+1}}=\delta_{X}^{k}$.

Another generalization is to consider fillings of chains by cycles, with volume given by $\|\cdot\|_{1}$-norm. Gromov [9] defined the filling volume function $F V$ of a 
manifold by using Lipschitz cycles; we will use cellular cycles. For $\alpha \in Z_{k-1}(X ; \mathbb{Z})$ a $(k-1)$-cycle, define

$$
F V_{X, \mathbb{K}}^{k}(\alpha)=\inf \left\{\|\beta\|_{1} \mid \beta \in C_{k}(X ; \mathbb{K}), \partial \beta=\alpha\right\}
$$

for $\mathbb{K}=\mathbb{R}$ or $\mathbb{K}=\mathbb{Z}$, and define the $k$-dimensional filling volume function of $X$ by

$$
F V_{X, \mathbb{K}}^{k}(n)=\sup _{\substack{\alpha \in Z_{k-1}(X ; \mathbb{Z}) \\\|\alpha\|_{1} \leq n}} F V_{X, \mathbb{K}}^{k}(\alpha) .
$$

As with $\delta^{M}$, we can specify the manifold to fill. If $N$ is a closed orientable $(k-1)$-dimensional manifold, we define

$$
F V_{X, \mathbb{K}}^{N}(n)=\sup _{\substack{\alpha: N \rightarrow X \\ \text { vol } \alpha \leq n}} F V_{X, \mathbb{K}}^{k}(\hat{\alpha}),
$$

where $\alpha$ is assumed to be admissible. Then if $(M, \partial M)$ is a compact orientable manifold pair with $\operatorname{dim}(M)=k$,

$$
F V_{X, \mathbb{Z}}^{\partial M}(n) \preceq \delta_{X}^{M}(n), \quad F V_{X, \mathbb{K}}^{\partial M}(n) \preceq F V_{X, \mathbb{K}}^{k}(n) .
$$

Finally, we define the filling size of a curve. The size of a chain is a notion from geometric measure theory which counts the number of distinct cells in the support of a chain. The filling size FSize describes the infimal size of a chain filling a curve. If $\beta=\sum_{i=1}^{r} b_{i} \Delta_{i} \in C_{k}(X ; \mathbb{K})$ for $b_{i} \neq 0 \in \mathbb{K}$ and distinct $k$-cells $\Delta_{i}$ of $X$, let size $\beta=r$. Let the support supp $\beta$ of $\beta$ be the minimal subcomplex of $X$ containing the $\Delta_{i}$. Define the filling size FSize $(\alpha)$ of an admissible loop $\alpha: S^{1} \rightarrow X$ by

$$
\begin{aligned}
& \operatorname{FSize}_{X}(\alpha)=\min \left\{\operatorname{size} \beta \mid \beta \in C_{2}(X ; \mathbb{R}), \partial \beta=\hat{\alpha}\right\}, \\
& \operatorname{FSize}_{X}(n)=\sup _{\substack{\alpha: S^{1} \rightarrow X \\
\operatorname{vol} \alpha \leq n}} \operatorname{FSize}_{X}(\alpha) .
\end{aligned}
$$

This represents the number of different 2-cells of $X$ necessary to support a filling of a loop.

Like $\delta$, the functions FSize and $F V$ are defined in terms of a CW-complex $X$, but can also be defined up to (1) for a group

Lemma 1. Let $k \geq 1$ and let $X_{1}$ and $X_{2}$, be $k$-connected $C W$-complexes such that $G$ acts on $X_{i}$ cocompactly, properly discontinuously, and by automorphisms. Let $\mathbb{K}=\mathbb{R}$ or $\mathbb{K}=\mathbb{Z}$. Then FSize $_{X_{1}} \sim$ FSize $_{X_{2}}$ and $F V_{X_{1}, \mathbb{K}}^{k+1} \sim F V_{X_{2}, \mathbb{K}}^{k+1}$.

Proof. It is enough to show that $\mathrm{FSize}_{X_{1}} \precsim \mathrm{FSize}_{X_{2}}$ and $F V_{X_{1}, \mathbb{K}}^{k+1} \precsim F V_{X_{2}, \mathbb{K}}^{k+1}$; the lemma then follows by symmetry.

By the Švarc-Milnor Lemma, $X_{1}$ and $X_{2}$ are both quasi-isometric to $G$, and thus are quasi-isometric. By Lemmas 12 and 13 of [2], there are cellular quasiisometries $f: X_{1}^{(k+1)} \rightarrow X_{2}^{(k+1)}$ and $g: X_{2}^{(k+1)} \rightarrow X_{1}^{(k+1)}$ and a cellular homotopy 
$h: X_{1}^{(k)} \times[0,1] \rightarrow X_{1}^{(k+1)}$ such that for all $x \in X_{1}^{(k)}, h(x, 0)=(g \circ f)(x)$ and $h(x, 1)=x$. Furthermore, there is a $c>0$ such that if

$$
f_{*}: C_{*}\left(X_{1}^{(k+1)} ; \mathbb{K}\right) \rightarrow C_{*}\left(X_{2}^{(k+1)} ; \mathbb{K}\right)
$$

is the map induced by $f$, then, for all $\sigma \in C_{i}\left(X_{1}^{(k+1)} ; \mathbb{K}\right)$, we have

$$
\left\|f_{*}(\sigma)\right\|_{1} \leq c\|\sigma\|_{1}, \quad \operatorname{size}\left(f_{*}(\sigma)\right) \leq c \operatorname{size}(\sigma) .
$$

Similar inequalities hold when $f$ is replaced by $g$ or $h$.

If $\alpha \in Z_{k}\left(X_{1} ; \mathbb{K}\right)$, then $f_{*}(\alpha)$ is a cycle in $X_{2}$, and there is a $(k+1)$-chain $\beta \in C_{k+1}\left(X_{2} ; \mathbb{K}\right)$ such that $\partial \beta=f_{*}(\alpha)$ and $\|\beta\|_{1} \leq F V_{X_{2}, \mathbb{K}}^{k+1}\left(f_{*}(\alpha)\right)+1$. Then

$$
\beta^{\prime}=g_{*}(\beta)+h_{*}(\alpha \times[0,1])
$$

has boundary $\alpha$ and

$$
F V_{X_{1}, \mathbb{K}}^{k+1}(\alpha) \leq\left\|\beta^{\prime}\right\|_{1} \leq c F V_{X_{2}, \mathbb{K}}^{k+1}\left(f_{*}(\alpha)\right)+c+c\|\alpha\|_{1} \leq c F V_{X_{2}, \mathbb{K}}^{k+1}\left(c\|\alpha\|_{1}\right)+c+c\|\alpha\|_{1} .
$$

Thus $F V_{X_{1}, \mathbb{K}}^{k+1} \precsim F V_{X_{2}, \mathbb{K}}^{k+1}$. Similarly, choosing $\beta$ so that $\operatorname{size}(\beta)=\operatorname{FSize}_{X_{2}}\left(f_{*}(\alpha)\right)$ shows that FSize $_{X_{1}, \mathbb{K}} \precsim$ FSize $_{X_{2}, \mathbb{K}}$.

If $G$ acts on $X$ in this way, we can define FSize $_{G}=$ FSize $_{X}$ and $F V_{G, \mathbb{K}}^{*}=F V_{X, \mathbb{K}}^{*}$, and these functions are well defined up to the equivalence relation (1). For all of these functions, we will omit the group when there is no confusion.

We will use FSize to provide a lower bound on some higher-dimensional filling volumes.

Theorem 2. If a group $G$ is $\mathfrak{F}^{k+1}$, then

$$
F V_{G^{k}, \mathbb{Z}}^{\left(S^{1}\right)^{k}}(n) \succeq \operatorname{FSize}_{G}\left(n^{1 / k}\right) .
$$

Proof. Let $X$ be the $(k+1)$-skeleton of the universal cover of a $K(G, 1)$ with finite $(k+1)$-skeleton, so that $X$ is $k$-connected and $G$ acts cocompactly, properly discontinuously, and by automorphisms on $X$. Let $\gamma: S^{1} \rightarrow X$ be an admissible map such that vol $\gamma=n$ and $\operatorname{FSize}_{X}(\gamma)=\operatorname{FSize}_{X}(n)$. Define the map $\alpha=$ $\gamma^{k}:\left(S^{1}\right)^{k} \rightarrow X^{k}$, where $X^{k}$ is given the product CW-structure; then $\operatorname{vol}(\alpha)=n^{k}$. Let $\beta \in C_{k+1}\left(X^{k} ; \mathbb{Z}\right)$ be a chain whose boundary is $\hat{\alpha}$ and such that

$$
\|\beta\|_{1}=F V_{X^{k}, \mathbb{Z}}^{\left(S^{1}\right)^{k}}(\alpha)
$$

Let $p_{1}, \ldots, p_{k}: X^{k} \rightarrow X$ be the maps projecting to each factor. Then $p_{i}(\operatorname{supp}(\beta))$ is a subcomplex of $X$ for each $i$. We claim that for some $i$, this subcomplex supports a 2-chain filling $\hat{\gamma}$ and thus has at least $\operatorname{FSize}_{X}(\gamma)$ cells. 
We proceed by contradiction, assuming that $\hat{\gamma}$ is not a boundary (over $\mathbb{R}$ ) in any of the $p_{i}(\operatorname{supp} \beta)$ 's. Then $\hat{\gamma}$ represents a non-zero element of $H_{1}\left(p_{i}(\operatorname{supp} \beta) ; \mathbb{R}\right)$ and by the universal coefficient theorem, there is a cohomology class $v_{i} \in H^{1}\left(p_{i}(\operatorname{supp} \beta) ; \mathbb{R}\right)$ such that $v_{i}(\hat{\gamma})=1$. Let $w_{i}=p_{i}^{*}\left(v_{i}\right) \in H^{1}(\operatorname{supp} \beta ; \mathbb{R})$. We claim that

$$
\left[\bigcup_{i=1}^{k} w_{i}\right](\hat{\alpha}) \neq 0
$$

this contradicts the fact that $\partial \beta=\hat{\alpha}$.

The $w_{i}$ pull back under $\alpha$ to the standard generators of $H^{1}\left(\left(S^{1}\right)^{k} ; \mathbb{R}\right)$, so their cup product is a class in $H^{k}(\operatorname{supp} \beta ; \mathbb{R})$ which pulls back to a generator $t$ of $H^{k}\left(\left(S^{1}\right)^{k} ; \mathbb{R}\right)$. Therefore,

$$
\left[\bigcup_{i=1}^{k} w_{i}\right](\hat{\alpha})
$$

is equal to $t$ evaluated on the fundamental class of $\left(S^{1}\right)^{k}$, and is thus non-zero. Since $\hat{\alpha}$ is a boundary in $\operatorname{supp} \beta$, this is impossible; any class in $H^{k}(\operatorname{supp} \beta ; \mathbb{R})$ must evaluate to 0 on $\hat{\alpha}$. Thus $\gamma$ is a boundary in $p_{i}(\operatorname{supp} \beta)$ for some $i$.

This implies that $p_{i}(\operatorname{supp} \beta)$ contains at least $\operatorname{FSize}_{X}(\gamma)$ 2-cells. Each of these is the image of a cell of $\operatorname{supp} \beta$, so $\operatorname{supp} \beta$ contains at least $\operatorname{FSize}_{X}(\gamma)$ cells. By the definition of a CW-complex, any $(k+1)$-cell of $X^{k}$ is contained in a finite subcomplex of $X^{k}$. Since there are only finitely many equivalence classes of cells of $X^{k}$ under the action of $G^{k}$, there is a constant $c>0$ such that for all $\sigma \in C^{*}\left(X^{k} ; \mathbb{R}\right)$, the number of cells in supp $\sigma$ is at most $c \operatorname{size} \sigma$. Thus

$$
F V_{X^{k}, \mathbb{Z}}^{\left(S^{1}\right)^{k}}(\alpha)=\|\beta\|_{1} \geq \operatorname{size} \beta \geq c^{-1} \operatorname{FSize}_{X}(\gamma),
$$

so

$$
F V_{X^{k}, \mathbb{Z}}^{\left(S^{1}\right)^{k}}\left(n^{k}\right) \geq c^{-1} \operatorname{FSize}_{X}(n)
$$

as desired.

Lemma 3. There is an aspherical group $G$ for which $\operatorname{FSize}(n)$ is not subrecursive.

Proof. Collins and Miller [6] constructed a group $G$ with unsolvable word problem and an aspherical presentation. This group is constructed from a free group by applying three successive $\mathrm{HNN}$-extensions where the associated subgroups are finitely generated free groups. This group belongs to the hierarchy $\mathscr{F}$ constructed by Gersten [8], and Theorem 4.3 of [8] states that for groups in this hierarchy, $\delta_{G}$ is bounded by a recursive function of $F V_{G, \mathbb{R}}^{S^{1}}$. Since $G$ has unsolvable word problem, $\delta_{G}$ is not subrecursive, so $F V_{G, \mathbb{R}}^{S^{1}}$ is also not subrecursive.

We claim that $F V_{G, \mathbb{R}}^{S^{1}}(n)$ is bounded by a recursive function of $\operatorname{FSize}_{G}(n)$ and thus that $\operatorname{FSize}_{G}(n)$ is not subrecursive. Let $X$ be the CW-complex corresponding to a finite aspherical presentation of $G$, let $c$ be the total length of the relators in the 
presentation and let $\alpha$ be a loop in $X$. Let $\beta \in C_{2}(\operatorname{supp} \beta ; \mathbb{R})$ be a 2-chain such that $\partial \beta=\alpha$ and such that $\operatorname{size}(\beta)=\operatorname{FSize}_{X}(\alpha)$. It suffices to show that there is a 2 -chain $\gamma$ such that $\partial \gamma=\alpha$ and $\|\gamma\|_{1}$ is bounded by a recursive function of size $\beta$.

Note that

$$
\operatorname{dim} C_{2}(\operatorname{supp} \beta ; \mathbb{R})=\operatorname{size} \beta, \quad \operatorname{dim} C_{1}(\operatorname{supp} \beta ; \mathbb{R}) \leq c \operatorname{size} \beta
$$

and $\partial: C_{2}(\operatorname{supp} \beta ; \mathbb{R}) \rightarrow C_{1}(\operatorname{supp} \beta ; \mathbb{R})$ is a linear map. The 2-cells of $\operatorname{supp} \beta$ correspond to a basis of $C_{2}(\operatorname{supp} \beta ; \mathbb{R})$ and the 1 -cells correspond to a basis of $C_{1}(\operatorname{supp} \beta ; \mathbb{R})$. In these bases, the equation $\partial \gamma=\alpha$ corresponds to a system of at most $c$ size $\beta$ linear equations in size $\beta$ variables with integer coefficients between $-c$ and $c$. Since $\beta$ is a solution, the system is solvable, and since such a system can be solved algorithmically, there is a solution whose $\|\cdot\|_{1}$ norm is bounded by a recursive function of size $\beta$. Thus $F V_{X, \mathbb{R}}^{S^{1}}(n)$ is bounded by a recursive function of $\operatorname{FSize}_{X}(n)$ and so $\operatorname{FSize}_{G}(n)$ is not subrecursive.

Using Theorem 2 and the following theorem of Brady et al. [3], Remark 2.6 (4), we can give lower bounds on filling functions of products of $G$ :

Theorem 4. If $\operatorname{dim} M=k+1 \geq 4$, then $\delta^{M} \leq \delta^{k}$ provided $\partial M$ is connected or $\delta^{k}$ is superadditive.

Corollary 5. For the group $G$ in Lemma $3, F V_{G^{k}, \mathbb{Z}}^{k+1}(n)$ is not subrecursive for $k \geq 1$ and $\delta_{G^{k}}^{k}(n)$ is not subrecursive for $k \geq 3$.

Note that the spheres with large filling volumes may be extremely distorted. If $\alpha:\left(S^{1}\right)^{k} \rightarrow X^{k}$ is as in the proof of Theorem 2 , then the construction in the proof of Theorem 4 results in a map $\alpha^{\prime}$ whose image contains non-recursively large 2-discs filling curves in the image of $\alpha$. These discs do not add to the $k$-volume of $\alpha^{\prime}$, but they increase its "complexity"; for instance, the Lipschitz constants of $\alpha$ and the number of simplices in a simplicial approximation grow non-recursively with vol $\alpha$. Brady's et al. theorem suggests that considering fillings of spheres is not particularly restrictive in high dimensions, since low-volume, high-complexity spheres can be used to approximate arbitrary manifolds. To study differences in filling different manifolds, it may be worthwhile to study other filling functions.

Finally, a theorem of Papasoglu [10] states that $\delta_{G}^{2}$ is a subrecursive function for any group $G$ which is $\mathcal{F}^{3}$. Combining this with Corollary 5, we obtain the following:

Corollary 6. There is a group $G$ such that $F V_{G, \mathbb{Z}}^{3}(n) \nsim \delta_{G}^{2}(n)$.

There are also examples of such groups with solvable word problem. To construct one such example, we let $G=\operatorname{BS}(1,2)$ and consider $G \times G$. One method to show that $\delta_{G}(n) \succeq 2^{n}$ uses the asphericity of a certain 2-dimensional $K(G, 1)$ to show that 
discs filling certain curves must contain an exponentially large number of 2-cells [7], 7.4. This method also shows that $\operatorname{FSize}_{G}(n) \succeq 2^{n}$ and thus, by Theorem 2 ,

$$
F V_{G \times G, \mathbb{Z}}^{3}(n) \succeq 2^{\sqrt{n}} .
$$

On the other hand, since there is a 2-dimensional $K(G, 1)$,

$$
\delta_{G}^{2}(n) \preceq n,
$$

and by Theorem 5.3 of [1],

$$
\delta_{G \times G}^{2}(n) \preceq n^{2} .
$$

In these groups, filling a torus takes substantially more volume than filling a sphere largely because the fundamental group of the torus is nontrivial. It would be interesting to see if there are other ways that the topology of the boundary affects the difficulty of filling. In particular, it remains open to find examples of groups in which filling a genus $g$ surface is harder than filling a torus.

The author would like to thank Hanna Bennett, Max Forester, and the referee for their comments on drafts of this paper, and NYU for its hospitality during part of the preparation of this paper.

\section{References}

[1] J. M. Alonso, W. A. Bogley, R. M. Burton, S. J. Pride, and X. Wang, Second order Dehn functions of groups. Quart. J. Math. Oxford (2) 49 (1998), 1-30. Zbl 0913.20020 MR 1617319

[2] J. M. Alonso, X. Wang, and S. J. Pride, Higher-dimensional isoperimetric (or Dehn) functions of groups. J. Group Theory 2 (1999), 81-112. Zbl 0927.20021 MR 1670329

[3] N. Brady, M. R. Bridson, M. Forester, and K. Shankar, Snowflake groups, PerronFrobenius eigenvalues and isoperimetric spectra. Geom. Topol. 13 (2009), 141-187. Zbl 05530178 MR 2469516

[4] M. R. Bridson, The geometry of the word problem. In Invitations to geometry and topology, Oxf. Grad. Texts Math. 7, Oxford Univ. Press, Oxford 2002, 29-91. Zbl 0996.54507 MR 1967746

[5] M. R. Bridson, Polynomial Dehn functions and the length of asynchronously automatic structures. Proc. London Math. Soc. (3) 85 (2002), 441-466. Zbl 1046.20027 MR 1912057

[6] D. J. Collins and C. F. Miller, III, The word problem in groups of cohomological dimension 2. In Groups St. Andrews 1997 in Bath, I, London Math. Soc. Lecture Note Ser. 260, Cambridge Univ. Press, Cambridge 1999, 211-218. Zbl 1001.20030 MR 1676618

[7] D. B. A. Epstein, J. W. Cannon, D. F. Holt, S. V. F. Levy, M. S. Paterson, W. P. Thurston, Word processing in groups. Jones and Bartlett Publishers, Boston 1992. Zbl 0764.20017 MR 1161694 
[8] S. M. Gersten, Homological Dehn functions and the word problem. http://www.math.utah.edu/ sg/Papers/df9.ps.gz

[9] M. Gromov, Geometric group theory (Sussex, 1991), vol. 2: Asymptotic invariants of infinite groups. London Math. Soc. Lecture Note Ser. 182, Cambridge University Press, Cambridge 1993. Zbl 0841.20039 MR 1253544

[10] P. Papasoglu, Isodiametric and isoperimetric inequalities for complexes and groups. $J$. London Math. Soc. (2) 62 (2000), 97-106. Zbl 0962.20032 MR 1771853

[11] R. Young, Filling inequalities for nilpotent groups. Preprint 2006. arXiv:math/0608174

Received June 5, 2008; revised July 1, 2009

R. Young, Institut des Hautes Études Scientifiques, Le Bois Marie, 35 route de Chartres, 91440 Bures-sur-Yvette, France,

and

Courant Institute of Mathematical Sciences 251 Mercer St. New York, NY 10012, U.S.A.

E-mail: rjyoung@ihes.fr;rjyoung1729@gmail.com 